\title{
A NOTE ON SPLITTING IN SOLVABLE GROUPS ${ }^{1}$
}

\author{
ERNEST E. SHULT
}

1. Introduction. The theorem presented below generalizes theorems of E. Schenkman [5] and G. Higman [4] concerning splitting in finite solvable groups. This generalization is achieved by applying results from the theory of formations recently developed by $\mathrm{W}$. Gaschütz [2], [3]. All groups considered here are finite and solvable. A formation, $\mathfrak{F}$, is a collection of groups closed under taking homomorphisms and subdirect products. It follows that every group, $G$, contains a characteristic subgroup, $G_{\mathfrak{F}}$, minimal with respect to the property that $G / G_{\mathfrak{F}} \in \mathfrak{F}$. A formation, $\mathfrak{F}$, is called saturated, if $G / \phi(G) \in \mathfrak{F}$ implies $G \in \mathfrak{F}$ for all $G$ (see [3]). $F$ is called an $\mathfrak{F}$-subgroup of $G$ if $F \in \mathfrak{F}$ and if $F \subseteq H \subseteq G$ implies $F H_{\mathfrak{F}}=H$. A theorem of Gaschütz [2], states that if $\mathfrak{F}$ is saturated, $\mathfrak{F}$-subgroups of $G$ always exist and are conjugate in $G$.

THEOREM. Let $\mathfrak{F}$ be a saturated formation and suppose that for a finite solvable group, $G, G_{\mathfrak{F}}$ is abelian. Then:

(i) the $\mathfrak{F}$-subgroups of $G$ complement $G_{\mathfrak{F}}$.

(ii) all complements of $G_{\mathfrak{\xi}}$ in $G$ are conjugate and hence are $\mathfrak{F}$-subgroups of $G$.

Let $L_{0}(G)=G$ and let $L_{i}(G)$ be the $i$ th term of the lower nilpotent series of $G$. If $\mathfrak{F}$ denotes the formation of groups having nilpotent length $\leqq k-1$, the theorem yields a theorem of Higman [4] which states that if $L_{k}(G)$ is abelian, $G$ splits over $L_{k}(G)$ and all complements of $L_{k}(G)$ in $G$ are conjugate. (This statement becomes a theorem of Schenkman [5] when $k=2$.) $\mathrm{R}$. Carter [1] was able to identify the complements in Higman's theorem as the relative system normalizers of $L_{k-1}(G)$ in $G$. From (ii) we may also identify them as the $\mathfrak{F}$ subgroups of $G$ (or as the Carter subgroups of $G$ when $k=2$ ). Our theorem yields a number of other interesting results on splitting when $\mathfrak{F}$ ranges over various saturated formations, for example, groups having nilpotent commutator subgroups, supersolvable groups, groups $G$, for which $G / G^{\prime}$ is a $\pi$-group, etc.

2. Preliminary results and proof of the theorem. Our proof employs a number of basic results of Gaschütz. First if $F$ is an $\mathfrak{F}$-subgroup

Received by the editors March 1, 1965.

1 This work was supported by a National Science Foundation grant while the author was at the University of Illinois, Summer, 1964. 
of $G, F \subseteq H \subseteq G$ implies that $F$ is an $\mathfrak{F}$-subgroup of $H$. Also if $N$ is normal in $G, F N / N$ is an $\mathfrak{F}$-subgroup of $G / N$ and every $\mathfrak{F}$-subgroup of $G / N$ has the form $F_{0} N / N$ where $F_{0}$ is an $\mathfrak{F}$-subgroup of $G$. Let $\pi$ and $\pi^{\prime}$ denote a partition of the set of primes. $O_{\pi^{\prime}}(G)$ denotes the maximal normal $\pi^{\prime}$-subgroup of $G$ and $O_{\pi^{\prime} x}(G)$ denotes the subgroup of $G$ for which $O_{\pi^{\prime} \pi}(G) / O_{\pi^{\prime}}(G)$ is the maximal normal $\pi$-subgroup of $G / O_{\pi^{\prime}}(G)$. A formation, $\mathfrak{F}$, is said to be locally defined if for some sequence of (possibly empty) formations, $f(p), p$ ranging over the primes, $G \in \mathfrak{F}$ if and only if $p \nmid|G|$ when $f(p)$ is empty and $G / O_{p^{\prime} p}(G)$ $\in \mathfrak{f}(p)$ otherwise. Gaschütz proved [2] that all locally defined formations are saturated and recently has announced ${ }^{2}$ the important result that, conversely, all saturated formations are locally defined by some sequence of local formations $\{f(p)\}$.

Proof of THE THEOREM. (i) If $G_{\mathfrak{F}}=E, G$ is its own $\mathfrak{F}$-subgroup and the theorem is trivial. Suppose then that $G_{\mathfrak{F}} \neq E$. Let $F$ be an $\mathfrak{F}$ subgroup of $G$. Since $F G_{\mathfrak{F}}=G$, to prove (i) it suffices to show that $F \cap G_{\mathfrak{F}}=E$. Suppose $F \cap G_{\S} \neq E$. Then since $G_{\mathfrak{F}}$ is abelian, $F \cap G_{\mathfrak{F}}$ is normal in $G$. Let $N$ be a minimal normal subgroup of $G$. Then $(G / N)_{\mathfrak{F}}=G_{\mathfrak{F}} N / N$ is abelian and $F N / N$ is an $\mathfrak{F}$-subgroup of $G / N$. By induction, $F N \cap G_{\mathfrak{F}} N \subseteq N$. Thus $F \cap G_{\mathfrak{F}}$ lies in every minimal normal subgroup of $G$. It follows that $F \cap G_{\Im}=N_{0}$, is the unique minimal normal subgroup of $G$.

Suppose $N_{0}=G_{\mathfrak{F}}$. Then $G=F N_{0}=F \in \mathfrak{F}$ whence $G_{\mathfrak{F}}=E$, a contradiction. Hence $N_{0} \subset G_{\mathfrak{F}}$.

Suppose $F \subset H \subset G$. Then $H G_{\mathfrak{F}}=G$ and so $G / G_{\Re} \simeq H /\left(H \cap G_{\mathfrak{F}}\right) \in \mathfrak{F}$ whence $H_{\mathfrak{F}} \subset G_{\mathfrak{F}}$. Since $H_{\mathfrak{F}}$ is now forced to be abelian and $F$ is an $\mathfrak{F}$-subgroup of $H, F \cap H_{\mathfrak{F}}=E$ by induction on $H$. On the other hand the fact that $G_{\mathfrak{F}}$ is abelian implies $H_{\mathfrak{F}}$ is normal in $G$ and hence $H_{\mathfrak{F}} \cap F$ contains $N_{0}$, a contradiction. Thus $F$ is maximal in $G$ and $G_{\S} / N_{0}$ and $N_{0}$ are successive chief factors of $G$. From the uniqueness of $N_{0}, G_{\mathfrak{F}}$ is an abelian p-group.

Let $Q$ be a $p^{\prime}$-subgroup of $G$ such that $Q G_{\Im}$ is normal in $G$. Then, since $G_{\mathfrak{F}}$ is abelian, $G_{\mathfrak{F}}=C_{G_{\mathfrak{F}}}(Q) \times\left[Q, G_{ף}\right]$ where each component is normal in $G$. Suppose $\left[Q, G_{\S}\right] \neq G_{\mathfrak{F}}$. Then uniqueness of $N_{0}$ implies $C_{G_{\mathfrak{F}}}(Q)=G_{\Im}$ and $Q$ is then normal in $G$. Because of the uniqueness of $N_{0}, Q=E$. Thus if $Q G_{\S} \Delta G$ either $Q=E$ or $G_{\mathfrak{F}}=\left[Q, G_{\S}\right]$.

Choose $B_{q}$ so that $B_{q} / N_{0}=O_{q^{\prime} q}\left(G / N_{0}\right)$, and set $T_{q}=O_{q^{\prime} q}(F)$. We shall show that $T_{q} \subseteq B_{q}$.

Suppose $q \neq p$. Then $G_{\S} \subseteq O_{q^{\prime}}(G)$, and $T_{q} G_{\S}$ is $q$-nilpotent and nor$\mathrm{mal}$ in $G$. Hence $T_{q} G_{\mathfrak{F}} \subseteq O_{\mathbf{q}^{\prime} \mathbf{q}}(G) \subseteq B_{\underline{q}}$.

${ }^{2}$ Communicated at the Michigan Conference of Finite Groups, March, 1964. 
Suppose $q=p$. Set $Q_{0}=O_{p^{\prime}}(F)$. Since $Q_{0} G_{\Im}$ is normalized by both $F$ and $G_{\mathfrak{F}}$, it is normal in $G$. By a previous remark, if $Q_{0} \neq E, G_{\mathfrak{F}}$ $=\left[Q_{0}, G_{\mathfrak{F}}\right]$. But the latter is impossible since $Q_{0} \subseteq O_{p^{\prime}}(F)$ and $N_{0}$ $\subseteq O_{p}(F)$ imply $\left[Q, N_{0}\right]=E$. Thus $Q_{0}=E$. As a result, $T_{p}=O_{p}(F)$ and $T_{p} G_{\mathfrak{F}}$, being normalized by $F$ and $G_{\mathfrak{F}}$, lies in $O_{p}(G) \subseteq B_{p}$. Hence $T_{p} \subseteq B_{p}$.

Since $\mathfrak{F}$ is saturated, we may assume $\mathfrak{F}$ is locally defined by $\{f(p)\}$. Thus $F \in \mathfrak{F}$ implies $F / T_{q} \in \mathfrak{f}(q)$ for each prime $q$ dividing $|F|$. Since $T_{q} \subseteq B_{q}$, it follows that $G / B_{q} \simeq F /\left(F \cap B_{q}\right)$ is a homomorphic image of $F / T_{q}$. Thus $G / B_{q} \in \mathfrak{f}(q)$ for each prime, $q$, dividing $\left[G: N_{0}\right]$. Since $\mathfrak{F}$ is locally defined by $\{\mathfrak{f}(q)\}, G / N_{0} \in \mathfrak{F}$ whence $G_{\mathfrak{F}} \subseteq N_{0}$, a contradiction, and (i) is proved.

(ii) In proving the second part of the theorem it suffices to show that every complement, $K$, of $G_{\mathfrak{F}}$ in $G$, is an $\mathfrak{F}$-subgroup of $G$. Again, there is nothing to prove if $G_{\mathfrak{F}}=E$. We suppose that $G_{\mathfrak{F}} \neq E$. Let $K$ be an arbitrary complement of $G_{\mathfrak{F}}$ in $G$ and choose $N$ minimal normal in $G$ contained in $G_{\S}$. Then $K N \cap G_{\S}=\left(K \cap G_{\xi}\right) N=N$ so $K N / N$ is a complement of $G_{\mathfrak{F}} / N=(G / N)_{\mathfrak{F}}$ in $G / N$. By induction $K N / N$ is an F-subgroup of $G / N$ and so $K N=F N$ where $F$ is an $\mathfrak{F}$-subgroup of $G$.

Suppose $N \subset G_{\mathfrak{F}}$. Then $K N=F N \subset G$. Now from (i), $F \cap N \subseteq F \cap G_{\mathfrak{F}}$ $=E$ and so $F \subset F N$. Consequently, $(F N)_{\mathfrak{F}}$, being a nontrivial characteristic subgroup of $N$ must coincide with $N$. Since $K$ complements $(K N)_{\mathfrak{F}}=N$ in $K N$, induction on $K N$ yields that $K$ is an $\mathfrak{F}$-subgroup of $K N$. Since $F$ is an $\mathfrak{F}$-subgroup of $K N$ as well as $G, K$ and $F$ are conjugate in $K N$. Thus $K$ is an $\mathfrak{F}$-subgroup of $G$.

Suppose $N=G_{\mathfrak{F}}$. Then $K \in \mathfrak{F}$ and $K$ is maximal in $G$. Under these circumstances $K$ satisfies the defining properties of an $\mathfrak{F}$-subgroup of $G$.

\section{REFERENCES}

1. R. Carter, Splitting properties of soluble groups, J. London Math. Soc. 36 (1961), 89-94.

2. W. Gaschütz, Zur Theorie der endlichen auflösbaren Gruppen, Math. Z. 80 (1963), 300-305.

3. W. Gaschütz and U. Lubeseder, Kennzeichnung gesättigter Formationen, Math. Z. 82 (1963), 198-199.

4. G. Higman, Complementation of Abelian normal subgroups, Publ. Math. Debrecen 4 (1956), 455-458.

5. E. Schenkman, The splitting of certain solvable groups, Proc. Amer. Math. Soc. 6 (1955), 286-290.

SOUTHERN ILLINOIS UNIVERSITY 